\title{
Reciclagem de Vidro Laminado: Utilização dos Vidros de Baixa Granulometria como Carga Abrasiva na Formulação de Vernizes de Alto Tráfego para Pisos de Madeira
}

\author{
Isabella M. Vargas \\ Engenharia Metalúrgica e de Materiais, Escola Politécnica da USP, \\ Renner Sayerlack S.A. \\ Hélio Wiebeck \\ Engenharia Metalúrgica e de Materiais, Escola Politécnica da USP
}

\begin{abstract}
Resumo: O vidro laminado, especialmente o proveniente dos pára-brisas, é de difícil reciclagem após sua utilização, sobretudo o resíduo de vidro constituído de partículas pequenas (com baixa granulometria). $\mathrm{O}$ vidro muito fino se caracteriza como um resíduo, uma vez que não pode ser recuperado na indústria vidreira, pois nos grandes fornos de fusão de vidro evita-se a utilização deste material para impedir que ocorra o fenômeno de arraste de pó pela chaminé, bem como a deposição de vidro fundido nas paredes internas do forno e do regenerador ${ }^{[1]}$. Neste estudo duas granulometrias de vidro foram utilizadas - malha 200 e 325 mesh - na formulação de vernizes baseados num oligômero uretano-acrilado e usado para pintura e proteção de pisos de madeira. A cura desses vernizes foi por meio de radiação ultravioleta. Os resultados encontrados mostraram que a resistência à abrasão do verniz com a adição de vidro fino aumentou significativamente.
\end{abstract}

Palavras-chaves: Vidro laminado, pára-brisa, verniz, piso de madeira, abrasão.

\section{Recycling of Laminated Glass: The Use of Low Granulometry Glasses as Abrasive Filler in the Formulation of Varnishes for Wood Flooring Application}

Abstract: Windshields are difficult to recycle after use, especially the residue of fine glass (with small granulometry). This material is characterized as a residue since it cannot be recovered in the glass industry, because in the furnaces of glass fusion fine glass is avoided to prevent deposition on the internal walls of the furnaces and the drag of this fine powder through the chimney ${ }^{[1]}$. In this work two different granulometries of this fine glass - 200 mesh and 325 mesh - were used as a filler in a varnish based on a urethane-acrylate oligomer used to cover and protect wood flooring. The cure of this varnish was through ultraviolet radiation. The results showed increased abrasion resistance for the varnish with addition of the fine glass.

Keywords: Laminated glass, windshield, varnish, wood flooring, abrasion.

\section{Introdução}

Os vidros laminados são compostos pelo "sanduíche" vidro/PVB/vidro e seu preparo se dá pela colocação do filme de poli(vinil butiral) - PVB - entre duas ou mais chapas de vidro soda-cal. Este conjunto é levado à autoclave e submetido a pressões e temperaturas adequadas ${ }^{[2,3]}$. Os vidros laminados atualmente encontram aplicação não somente na indústria automobilística, na confecção dos pára-brisas, mas também são empregados na construção civil em portas e divisórias de vidro e em janelas sem esquadrias, por fator de segurança $\mathrm{a}^{[1]}$.

Atualmente o destino desses materiais é o aterro sanitário e só no Brasil são descartados cerca de $120 \mathrm{mil}$ pára-brisas/mês. Como cada um deles pesa $15 \mathrm{~kg}$ - sendo $14 \mathrm{~kg}$ de vidro e $1 \mathrm{~kg}$ de PVB -, aproximadamente 1,8 mil toneladas do produto termina mensalmente nos aterros, e se o cálculo for feito para um ano, encontra-se o valor de 21,6 mil toneladas de vidro laminado - lembrando que o PVB levaria 500 anos para ser assimilado pela natureza e o vidro é praticamente indestrutível ${ }^{[6]}$.

$\mathrm{O}$ vidro, por sua vez, com $72 \%$ de $\mathrm{SiO}_{2}$ em sua composição, tem alta dureza, além de durabilidade química e bom espectro de transmissão na região da luz visível ${ }^{[4,5]}$. No presente trabalho teve-se, portanto, o intuito de aproveitar esse material, especialmente os vidros de baixa granulometria - que não podem ser reaproveitados em fornos da indústria vidreira -, na fabricação de produtos utilizados para o revestimento e proteção de pisos de madeira, após a sua separação do filme de PVB por meio de moagens.

Os pisos de madeira são os preferidos em razão de sua estética, conforto e higiene. Entretanto, essa madeira deve ser protegida de manchas e ações do tempo, e para que isto ocorra é necessário que ela receba a aplicação de uma ca-

Autor para correspondência: Hélio Wiebeck, Engenharia Metalúrgica e de Materiais, Escola Politécnica da USP, Av. Prof. Lineu Prestes 580, Conj. das Químicas, BI.18, CEP 05508-900, São Paulo, SP, Brasil.E-mail: hwiebeck@usp.br 
mada protetora. Dentre os diversos tipos de revestimentos possíveis para os pisos de madeira, um dos mais utilizados industrialmente é o revestimento curado por radiação ultravioleta, com alta resistência química e à abrasão $0^{[7]}$, e é nesse tipo de revestimento que o vidro foi reaproveitado. Os vidros de baixa granulometria foram utilizados como cargas para aumentar a resistência ao desgaste dos vernizes alto tráfego, assim chamados por sua alta resistência à abrasão, e que são o "coração" do sistema de revestimento, pois tanto a sua espessura quanto a sua composição química determinarão importantes propriedades como resistência à abrasão, elasticidade, entre outras ${ }^{[8]}$.

Diferentes granulometrias foram testadas para análise da influência do tamanho da partícula nas propriedades do filme, bem como diferentes quantidades e combinações com outras cargas, como o óxido de alumínio, por exemplo, que, embora muito utilizado para conferir resistência à abrasão aos vernizes curados por radiação ultravioleta, diminui o tempo de vida dos equipamentos de aplicação dos vernizes de alto tráfego devido a sua alta abrasividade.

\section{Experimental}

Os vidros malha 200 e 325 mesh foram fornecidos pela Ekoglass Tesselos e Pastilhas de Vidro e são separados do vidro a ser reciclado por apresentar granulometria muito pequena. Para a separação do PVB e do vidro, a empresa Ekoglass criou um sistema de moinhos que não pode ser demonstrado neste trabalho por ser caracterizado como segredo industrial. Para a seleção do tamanho de partícula do vidro foram utilizadas peneiras com aberturas de 200 e 325 mesh na saída do moinho.

Para a preparação dos vernizes AT foi utilizada uma base cuja formulação encontra-se na Tabela 1.

Para essa base empregou-se uma resina uretano-acrilada difuncional, pois esse tipo de oligômero é o mais utilizado em vernizes para pisos de madeira devido à sua alta resistência mecânica, além de quase sempre ser flexível, com alta resistência química e boas propriedades de adesão ${ }^{[9]}$.

Como o oligômero utilizado apresenta alta viscosidade, foi escolhido como monômero acrílico da formulação o TPGDA (tripropileno glicol diacrilato) para a obtenção da

Tabela 1. Fórmula da base utilizada para a preparação dos vernizes AT.

\begin{tabular}{|c|c|}
\hline Quantidade de MP & Base para verniz AT(\%) \\
\hline Resina Uretano-Acrilada ${ }^{a}$ & 66,8 \\
\hline TPGDA $^{\mathrm{b}}$ & 25 \\
\hline Darocur $1173^{c}$ & 3 \\
\hline Byk $-080^{d}$ & 0,2 \\
\hline Gel de bentone $e^{e}$ & 5 \\
\hline
\end{tabular}

${ }^{a}$ Fabricada pela Renner Sayerlack S.A.; ${ }^{\text {TTPGDA (tripropileno glicol diacri- }}$ lato) fornecido pela UCB do Brasil; 'Fornecido pela Ciba Specialty Chemicals Inc.; ${ }^{\mathrm{d} A d i t i v o ~ a n t i-e s p u m a n t e ~ f o r n e c i d o ~ p e l a ~ B y k ~ C h e m i e ; ~ e ~}{ }^{\mathrm{e} O}$ gel de bentone apresenta a seguinte composição: $85 \%$ de xileno, $10 \%$ de bentone $\mathrm{SD}^{\circledR}-1$ (bentonita organofílica fornecida pela Elementis Specialties) e 5\% de álcool etílico. viscosidade adequada de aplicação. Esse monômero é muito usado para a obtenção de um bom balanço entre densidade de ligação cruzada, boa velocidade de cura e baixa viscosida$\mathrm{de}^{[10]}$. Como fotoiniciador optou-se pelo Darocur 1173, que é o mais indicado para a cura de produtos transparentes. O aditivo Byk 080 é um antiespumante e foi adicionado à formulação para evitar a formação de espuma durante a preparação do produto e também no momento da aplicação deste na máquina de rolo, e o gel de bentone foi adicionado para evitar a sedimentação das cargas utilizadas para aumentar a resistência à abrasão do verniz.

À base preparada conforme formulação da Tabela 1 foram adicionados os pós de vidros de baixa granulometria e as cargas comparativas (neste caso o óxido de alumínio e o agalmatolito) nas quantidades indicadas na Tabela 2. Para a adição das cargas, a base foi pesada no béquer e colocada em um agitador do tipo Dispermat e o pó foi adicionado sob agitação. Após a adição da carga, o produto foi agitado por 15 minutos a $1500 \mathrm{rpm}$.

Tabela 2. Verniz AT correspondente a cada tipo e quantidade de carga utilizada.

\begin{tabular}{|c|c|c|}
\hline Verniz AT & Carga(s) utilizada(s) & $\begin{array}{l}\text { Quantidade } \\
\text { de carga(\%) }\end{array}$ \\
\hline 1 & Sem carga & 0 \\
\hline 2 & Óxido de alumínio & 10 \\
\hline 3 & Agalmatolito & 10 \\
\hline 4 & Pó de vidro malha 200 & 5 \\
\hline 5 & Pó de vidro malha 200 & 10 \\
\hline 6 & Pó de vidro malha 200 & 15 \\
\hline 7 & Pó de vidro malha 200 & 20 \\
\hline 8 & Pó de vidro malha 200 & 25 \\
\hline 9 & Pó de vidro malha 200 & 30 \\
\hline 10 & Pó de vidro malha 325 & 5 \\
\hline 11 & Pó de vidro malha 325 & 10 \\
\hline 12 & Pó de vidro malha 325 & 15 \\
\hline 13 & Pó de vidro malha 325 & 20 \\
\hline 14 & Pó de vidro malha 325 & 25 \\
\hline 15 & Pó de vidro malha 325 & 30 \\
\hline 16 & $\begin{array}{l}\text { Vidro malha } 200 \text { + óxido } \\
\text { de alumínio }\end{array}$ & $\begin{array}{l}7 \text { e } 3 \\
\text { respectivamente }\end{array}$ \\
\hline 17 & $\begin{array}{l}\text { Vidro malha } 200 \text { + óxido } \\
\text { de alumínio }\end{array}$ & $\begin{array}{l}5 \text { e } 5 \\
\text { respectivamente }\end{array}$ \\
\hline 18 & $\begin{array}{l}\text { Vidro malha } 200 \text { + óxido } \\
\text { de alumínio }\end{array}$ & $\begin{array}{c}3 \text { e } 7 \\
\text { respectivamente }\end{array}$ \\
\hline 19 & $\begin{array}{l}\text { Vidro malha } 200 \text { + óxido } \\
\text { de alumínio }\end{array}$ & $\begin{array}{l}5 \text { e } 10 \\
\text { respectivamente }\end{array}$ \\
\hline 20 & $\begin{array}{l}\text { Vidro malha } 325 \text { + óxido } \\
\text { de alumínio }\end{array}$ & $\begin{array}{l}7 \text { e } 3 \\
\text { respectivamente }\end{array}$ \\
\hline 21 & $\begin{array}{l}\text { Vidro malha } 325 \text { + óxido } \\
\text { de alumínio }\end{array}$ & $\begin{array}{l}5 \text { e } 5 \\
\text { respectivamente }\end{array}$ \\
\hline 22 & $\begin{array}{l}\text { Vidro malha } 325 \text { + óxido } \\
\text { de alumínio }\end{array}$ & $\begin{array}{c}3 \text { e } 7 \\
\text { respectivamente }\end{array}$ \\
\hline 23 & $\begin{array}{l}\text { Vidro malha } 325 \text { + óxido } \\
\text { de alumínio }\end{array}$ & $\begin{array}{l}5 \text { e } 10 \\
\text { respectivamente }\end{array}$ \\
\hline
\end{tabular}


Na Tabela 2 são apresentadas as concentrações de carga utilizadas e a nomenclatura que será empregada neste trabalho para cada verniz AT.

O óxido de alumínio tipo TB-L 600, fornecido pela Elfusa, foi usado como padrão neste estudo, pois é a carga mais utilizada atualmente para a fabricação de vernizes AT, e o agalmatolito escolhido como comparativo foi o HTM20 fornecido pela Mineração Matheus Leme Ltda. O uso do agalmatolito neste estudo deveu-se ao fato de ele ser uma carga muito empregada na indústria de tintas e vernizes para madeira e apresentar quantidade considerável de $\mathrm{SiO}_{2}$, entre 51 e $64 \%$, além de conter de 28 a $36 \%$ de $\mathrm{Al}_{2} \mathrm{O}_{3}$.

Nos vernizes 16 ao 23 foram feitas misturas de pó de vidro com óxido de alumínio, pois alguns estudos obtiveram bons resultados de resistência à abrasão com a combinação desses dois materiais ${ }^{[11]}$.

Para os testes de resistência à abrasão os vernizes foram aplicados sobre peças de madeira Jatobá e o processo utilizado e as camadas aplicadas podem ser observados na Figura 1.

Todos os produtos foram aplicados em máquina de rolo. O isolante e o acabamento são produzidos pela Renner Sayerlack e as camadas aplicadas de cada um foram, respectivamente, 15 e $20 \mathrm{~g} \cdot \mathrm{m}^{-2}$. Cada camada de verniz de alto tráfego aplicada corresponde a $20 \mathrm{~g} . \mathrm{m}^{-2}$. O isolante e os vernizes foram geleificados com lâmpadas de mercúrio com dosagem de $161 \mathrm{~mJ} . \mathrm{cm}^{-2}$ e irradiância de $250 \mathrm{~mW} . \mathrm{cm}^{-2}$. Os vernizes não podem ser curados completamente, pois apresentam dificuldade para o lixamento devido à sua dureza; portanto, para a aderência entre camadas eles foram apenas geleificados. O acabamento foi curado com uma lâmpada de mercúrio, com uma dosagem de 544 mJ.cm ${ }^{-2}$ e uma irradiância de 254 mW.cm ${ }^{-2}$, para obtenção de cura total. Após a aplicação dos vernizes, as peças de Jatobá foram cortadas na medida de $10 \times 10 \mathrm{~cm}$ com um furo central de diâmetro de $6,3 \mathrm{~mm}$.

Para os ensaios de resistência à abrasão foram utilizadas duas normas: ASTM D 4060-01 e NEMA LD 3-13. Ambas determinam a resistência à abrasão de revestimentos orgânicos por meio do equipamento Taber Abraser, com a diferença que a norma ASTM utiliza como rebolo abrasivo o CS-17 e a norma NEMA, um rebolo de borracha neoprene (CS-0) revestido com uma lixa denominada $\mathrm{S}-42$.

Para a realização dos ensaios com o rebolo CS-17, em primeiro lugar, foi colocado sobre cada braço do equipamento o respectivo rebolo e ajustado o peso de cada braço. Neste caso foi utilizado um peso de $1000 \mathrm{~g}$ em cada braço.

Após a calibração dos rebolos com o disco abrasivo S-11, que também foi realizada a cada 500 ciclos, iniciou-se o des-

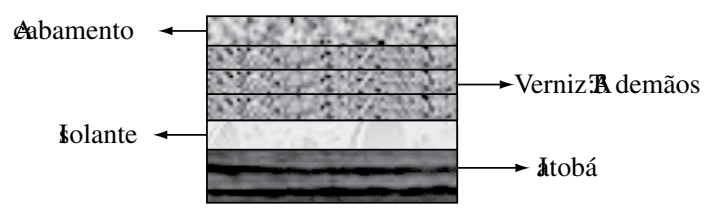

Figura 1. Processo aplicado para os testes de resistência à abrasão. gaste da peça e a cada 100 ciclos a mesma foi pesada em uma balança semi-analítica com resolução de $1 \mathrm{mg}$.

Para a observação do desgaste do revestimento aplicou-se um tingidor verde sobre o círculo desgastado pelos rebolos com o auxílio de um pedaço de algodão; após alguns minutos, o referido tingidor foi removido com um algodão embebido em acetona. Se o tingidor penetrar nas fibras da madeira e tingi-la, isto significa que o revestimento já foi removido; caso contrário, se o tingidor for completamente removido da peça sem deixar vestígios, significa que a película protetiva de verniz ainda permanece sobre a peça e que esta retornou para o equipamento para a continuação do desgaste. Esse procedimento foi repetido a cada 100 ciclos. Considerou-se o final do ensaio quando o tingimento foi percebido nos quatro quadrantes da peça.

Com os valores de cada pesagem das peças foi possível construir o gráfico número de ciclos $\mathrm{x}$ desgaste (em mg) para o cálculo da taxa de desgaste. Com os dados obtidos nas pesagens, a cada 100 ciclos, traçou-se a reta média no gráfico e a tangente da mesma é a taxa de desgaste. $\mathrm{O}$ valor de $\Delta \mathrm{m} / \Delta$ ciclos nada mais é do que o coeficiente angular da reta que foi calculado pelo software Microsoft ${ }^{\circledR}$ Excel quando da construção da reta média das curvas obtidas para cada verniz. O resultado foi multiplicado por 1000 para obter-se o valor da taxa de desgaste em $\mathrm{mg} / 1000$ ciclos.

Para a realização dos ensaios utilizando a lixa abrasiva $\mathrm{S}-42$, os mesmos procedimentos foram efetuados com as seguintes diferenças: utilizou-se o peso de $500 \mathrm{~g}$ em cada braço, pois a lixa $\mathrm{S}-42$ é bem mais abrasiva do que os rebolos CS-17; as pesagens das peças foram realizadas a cada 25 ciclos; a cada 250 ciclos aspirou-se o pó que ficou retido na lixa dos rebolos, e a cada 500 ciclos as lixas $\mathrm{S}-42$ foram trocadas. Outra diferença no caso do abrasivo S-42 é que o valor de taxa de desgaste encontrado foi multiplicado por 100 para obter-se o valor de taxa de desgaste em mg/100 ciclos.

\section{Resultados e Discussão}

Nos gráficos das Figuras 2 e 3 são mostrados os resultados dos testes de resistência à abrasão de cada verniz e para cada um deles foram construídos os gráficos do desgaste. As linhas de tendência (ou reta média), suas equações e os desvios-padrões $\left(\mathrm{R}^{2}\right)$ foram desenhados pelo software Microsoft ${ }^{\circledR}$ Excel.

Com os valores obtidos nas equações das curvas de tendência construídas pelo Excel foi possível ter a taxa de desgaste de cada verniz para os dois tipos de ensaios. Esses valores estão apresentados na Tabela 3 , bem como os valores de número de ciclos de cada ensaio.

Com os valores de taxa de desgaste e número de ciclos construiu-se um gráfico para cada tipo de abrasivo para melhor visualização dos resultados, os quais são mostrados na Figura 4.

Para os resultados com CS-17 observa-se pelo gráfico da Figura 4 que os vernizes que obtiveram maior resistência à 
Resistência à abrasão - 10\% de carga - CS17

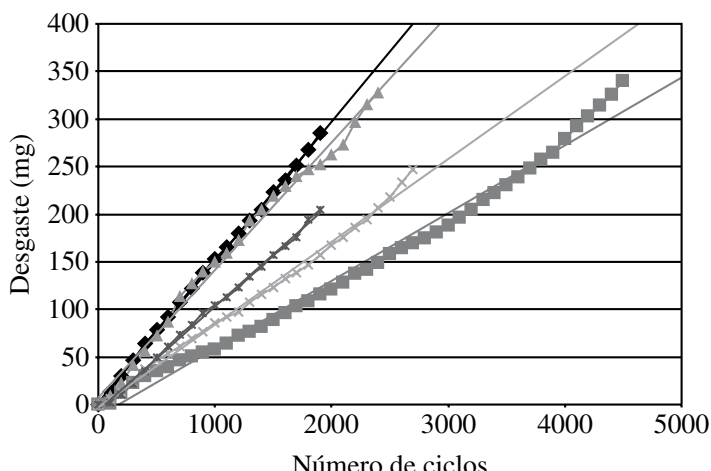

úmero de ciclos

\begin{tabular}{|ccc|}
$\rightarrow \sim$ Verniz 1 & - Verniz 3 & $\rightarrow$ Verniz 11 \\
$\mathrm{y}=0,1475 \mathrm{x}+2,4857$ & $\mathrm{y}=0,134 \mathrm{x}+7,6338$ & $\mathrm{y}=0,1096 \mathrm{x}-6,6$ \\
$\mathrm{R}^{2}=0,9993$ & $\mathrm{R}^{2}=0,9918$ & $\mathrm{R}^{2}=0,9975$ \\
- Verniz 2 & $*$ Verniz 5 & \\
$\mathrm{y}=0,0711 \mathrm{x}-12,185$ & $\mathrm{y}=0,087 \mathrm{x}-2,6773$ & \\
$\mathrm{R}^{2}=0,987$ & $\mathrm{R}^{2}=0,995$ & \\
\hline
\end{tabular}

Resistência à abrasão - 10\% de carga (misturas) - CS17

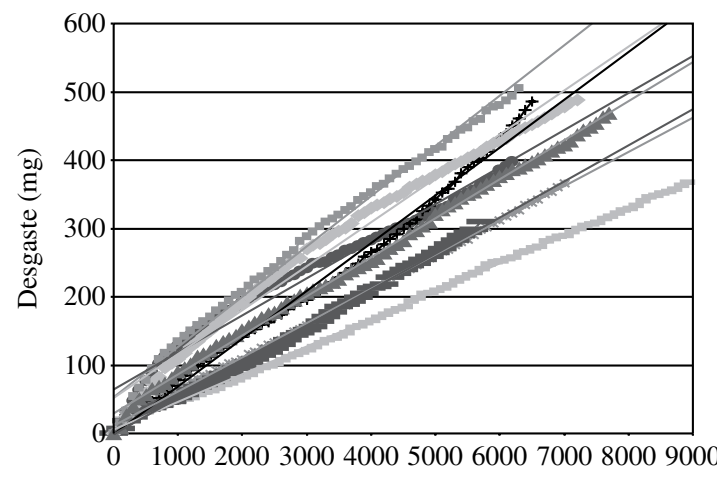

Número de ciclos

\begin{tabular}{|c|c|}
\hline $\begin{array}{c}-\% \text { Verniz } 16 \\
\mathrm{y}=0,0501 \mathrm{x}+10,969 \\
\mathrm{R}^{2}=0,9988\end{array}$ & $\begin{array}{c}-\bullet \text { Verniz } 17 \\
\mathrm{y}=0,0542 \mathrm{x}+64,896 \\
\mathrm{R}^{2}=0,9696\end{array}$ \\
\hline $\begin{array}{c}\text { +Verniz } 18 \\
\mathrm{y}=0,0699 \mathrm{x}-1,3826 \\
\mathrm{R}^{2}=0,9927\end{array}$ & $\begin{array}{c}\mathrm{y}=0,0406 \mathrm{x}+5,8347 \\
\mathrm{R}^{2}=0,9984\end{array}$ \\
\hline $\begin{array}{c}- \text { Verniz } 20 \\
\mathrm{y}=0,0528 \mathrm{x}-1,0929 \\
\mathrm{R}^{2}=0,9972\end{array}$ & $\begin{array}{c}\mathrm{y}=0,0642 \mathrm{x}+52,539 \\
\mathrm{R}^{2}=0,9824\end{array}$ \\
\hline $\begin{array}{c}\mathrm{y}=0,0735 \mathrm{x}+53,038 \\
\mathrm{R}^{2}=0,9905\end{array}$ & $\begin{array}{c}\mathrm{y}=0,0571 \mathrm{x}+29,867 \\
\mathrm{R}^{2}=0,9972\end{array}$ \\
\hline
\end{tabular}

Resistência à abrasão (idro \#00) - CS 17

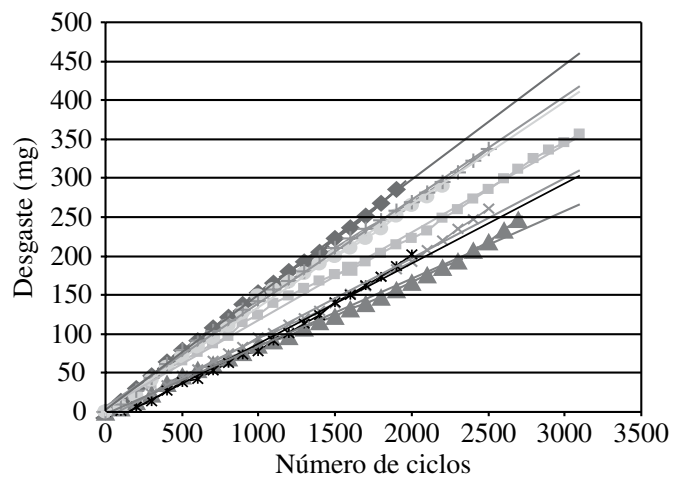

\begin{tabular}{|c|c|}
\hline $\begin{array}{c}-\sim \text { Verniz } 1 \\
\mathrm{y}=0,1475 \mathrm{x}+2,4857 \\
\mathrm{R}^{2}=0,9993\end{array}$ & $\begin{array}{cc}y=0,1125 x+5,8239 & y=0,087 x-2,6773 \\
\mathrm{R}^{2}=0,9974 & \mathrm{R}^{2}=0,995\end{array}$ \\
\hline 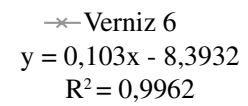 & $\begin{array}{cc}\rightarrow \text {-Verniz } 7 & - \text { Verniz } 8 \\
\mathrm{y}=0,1027 \mathrm{x}-14,81 & \mathrm{y}=0,1319 \mathrm{x}+2,8696 \\
\mathrm{R}^{2}=0,9905 & \mathrm{R}^{2}=0,9963\end{array}$ \\
\hline $\begin{array}{c}\text { +Verniz } 9 \\
\mathrm{y}=0,1327 \mathrm{x}+7,0769 \\
\mathrm{R}^{2}=0,9981\end{array}$ & \\
\hline
\end{tabular}

Resistência à abrasão (idro 25 ) - CS 17

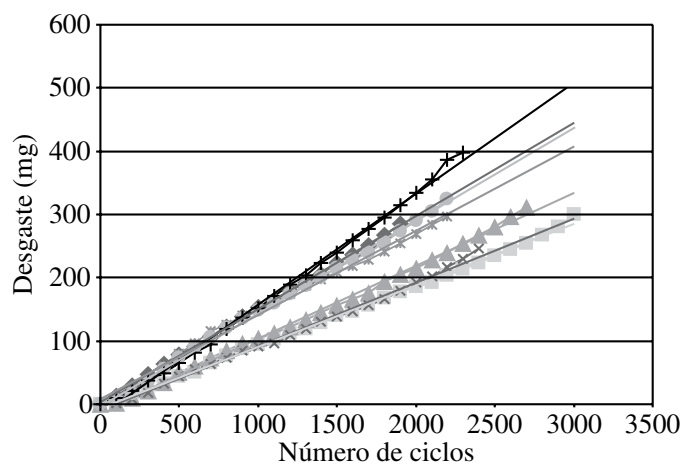

- Verniz 1 - Verniz $11 \quad *$ Verniz 13

$y=0,1475 x+2,4857 y=0,1149 x-10,658 y=0,1334 x+7,7935$

$\mathrm{R}^{2}=0,9993 \quad \mathrm{R}=0,9974 \quad \mathrm{R}^{2}=0,9913$

-Verniz $10 \quad *$ Verniz $12 \quad-$-Verniz 14

$y=0,0973 x-6,3427 \quad y=0,101 x-9,0892 \quad y=0,1465 x-2,7609$

$\mathrm{R}=0,9976 \quad \mathrm{R}=0,9957 \quad \mathrm{R}^{2}=0,9979$

+ Verniz 15

$\mathrm{y}=0,176 \mathrm{x}-18,547$

$\mathrm{R}=0,9962$

Figura 2. Gráficos de número de ciclos x taxa de desgaste para os vernizes utilizando o rebolo CS-17 como abrasivo.

abrasão, ou seja, menor taxa de desgaste, foram aqueles em que houve as combinações de óxido de alumínio e vidros, tanto \#325 quanto \#200, que são os vernizes 16 ao 23. É possível notar também que quanto maior a quantidade de vidro na mistura, maior a resistência à abrasão, o que foi observado no verniz 16 ao 18 (em que se utilizou o vidro \#200) e no verniz 20 ao 22 (em que se utilizou o vidro \#325). Resultados de sinergia entre o óxido de alumínio e sílicas já haviam sido observados onde a resistência à abrasão de um sistema poliacrílico aumentou em quase $100 \%$ com a combinação dos dois óxidos numa proporção de aproximadamente $1,2 \mathrm{de} \mathrm{SiO}_{2}$ : $1 \mathrm{de} \mathrm{Al}_{2} \mathrm{O}_{3}{ }^{[13]}$. 
Resistência à abrasão (vernizes com 10\% de carga) - S42

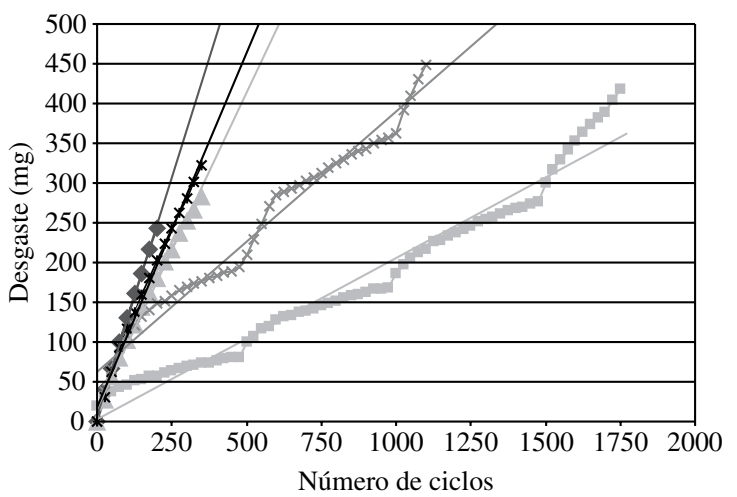

\begin{tabular}{|c|c|}
\hline $\begin{array}{c}- \text { Verniz } 1 \\
\mathrm{y}=1,1993 \mathrm{x}+7,0667 \\
\mathrm{R}^{2}=0,998\end{array}$ & $\begin{array}{c}\mathrm{y}=0,7941 \mathrm{x}+16,692 \\
\mathrm{R}^{2}=0,9925\end{array}$ \\
\hline $\begin{array}{c}\mathrm{y}=0,2028 \mathrm{x}+2,8134 \\
\mathrm{R}^{2}=0,9661\end{array}$ & $\begin{array}{c}* \text { Verniz } 5 \\
y=0,3271 x+62,692 \\
R^{2}=0,9715\end{array}$ \\
\hline \multicolumn{2}{|c|}{$\begin{array}{c}\text { *-Verniz } 11 \\
\mathrm{y}=0,8906 \mathrm{x}+18,483 \\
\mathrm{R}^{2}=0,9932\end{array}$} \\
\hline
\end{tabular}

Resistência à abrasão (vernizes com $10 \%$ de carga) - S42

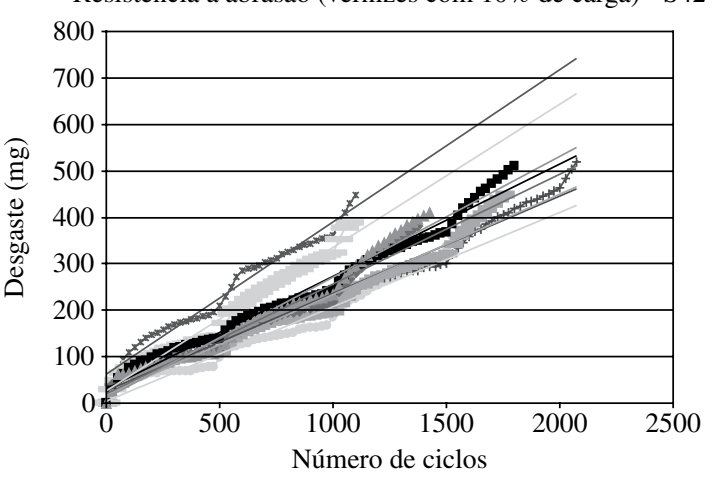

\begin{tabular}{|cc|}
\hline *-Verniz 16 & - - Verniz 17 \\
$y=0,3271 x+62,692$ & $y=0,2026 x+5,0538$ \\
$R^{2}=0,9715$ & $R^{2}=0,97$ \\
- Verniz 19 & + Verniz 18 \\
$y=0,2165 x+16,937$ & $y=0,2095 x+26,556$ \\
$R^{2}=0,9724$ & $R^{2}=0,9793$ \\
& \\
- Verniz 20 & - Verniz 21 \\
$y=0,3087 x+26,415$ & $y=0,2364 x+20,538$ \\
$R^{2}=0,9794$ & $R^{2}=0,9607$ \\
& \\
- Verniz 22 & - Verniz 23 \\
$y=0,242 x+31,223$ & $y=0,2599 x+11,614$ \\
$R^{2}=0,9823$ & $R^{2}=0,9688$
\end{tabular}

Resistência à abrasão (vidro \#00) - S42

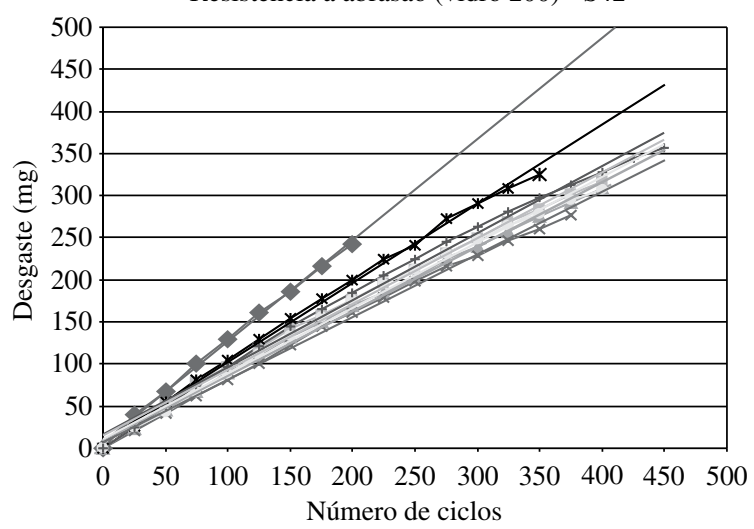

\begin{tabular}{|cc|}
\hline- Verniz 1 & - Verniz 4 \\
$y=1,1993 x+7,0667$ & $y=0,7793 x+15,255$ \\
$R^{2}=0,998$ & $R^{2}=0,9949$ \\
& \\
- Verniz 5 & $*$ Verniz 6 \\
$y=0,7654 x+10,157$ & $y=0,7452 x+6,5294$ \\
$R^{2}=0,9972$ & $R^{2}=0,9968$ \\
& \\
& \\
* Verniz 7 & Verniz 8 \\
0,9376x $+8,6583$ & $y=0,7788 x+10,647$ \\
$R^{2}=0,9973$ & $R^{2}=0,9941$ \\
+ Verniz 9 & \\
$y=0,7964 x+16,595$ & \\
$R^{2}=0,9933$ & \\
\hline
\end{tabular}

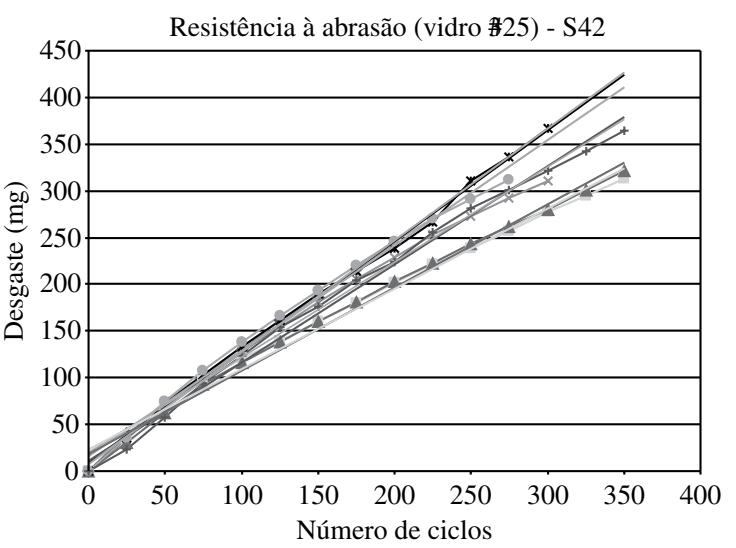

\begin{tabular}{|c|c|}
\hline $\begin{array}{c}\overrightarrow{\bullet-V e r n i z ~ 1} \\
=1,1993 x+7,0667 \\
\mathrm{R}^{2}=0,998\end{array}$ & $\begin{array}{c}\text { Verniz } 10 \\
\mathrm{y}=0,8587 \mathrm{x}+23,258 \\
\mathrm{R}^{2}=0,99\end{array}$ \\
\hline $\begin{array}{c}- \text { Verniz } 11 \\
=0,8906 x+18,483 \\
R^{2}=0,9932\end{array}$ & $\begin{array}{c}\text { * Verniz } 12 \\
\mathrm{y}=1,0198 \mathrm{x}+19,495 \\
\mathrm{R}^{2}=0,9908\end{array}$ \\
\hline $\begin{array}{c}\text { *-Verniz } 13 \\
=1,1892 x+8,3077 \\
R^{2}=0,9979\end{array}$ & $\begin{array}{c}\longrightarrow-\text { Verniz } 14 \\
\mathrm{y}=1,1266 \mathrm{x}+16,346 \\
\mathrm{R}^{2}=0,9919\end{array}$ \\
\hline $\begin{array}{l}\text { + Verniz } 15 \\
=1,0491 \mathrm{x}+11,333 \\
\mathrm{R}^{2}=0,9942\end{array}$ & \\
\hline
\end{tabular}

Figura 3. Gráficos de número de ciclos $x$ taxa de desgaste para os vernizes utilizando o rebolo $S-42$ como abrasivo. 
Vargas, I. M.; Wiebeck, H. - Reciclagem de vidro laminado

Tabela 3. Resultados de abrasão obtidos com os vernizes.

\begin{tabular}{|c|c|c|c|c|c|c|}
\hline \multirow[t]{2}{*}{ Verniz } & \multicolumn{3}{|c|}{ CS-17 } & \multicolumn{3}{|c|}{$S-42$} \\
\hline & $\begin{array}{l}\text { Tx. de desgaste } \\
\text { (mg/1000 ciclos) }\end{array}$ & $\mathbf{N}^{\circ}$ de ciclos & $\begin{array}{l}\text { Massa total } \\
(\mathrm{mg})\end{array}$ & $\begin{array}{l}\text { Tx. de desgaste } \\
\text { (mg/100 ciclos) }\end{array}$ & $\mathrm{N}^{\circ}$ de ciclos & $\begin{array}{l}\text { Massa total } \\
\quad(\mathbf{m g})\end{array}$ \\
\hline 1 & 147,5 & 1900 & 285 & 119,30 & 200 & 243 \\
\hline 2 & 71,1 & 4500 & 340 & 20,28 & 1800 & 424 \\
\hline 3 & 134,0 & 2400 & 328 & 79,41 & 350 & 283 \\
\hline 4 & 112,5 & 3100 & 356 & 77,93 & 400 & 317 \\
\hline 5 & 87,0 & 2700 & 247 & 32,71 & 400 & 309 \\
\hline 6 & 103,0 & 2500 & 261 & 74,52 & 375 & 277 \\
\hline 7 & 102,7 & 2000 & 202 & 93,76 & 350 & 325 \\
\hline 8 & 131,9 & 2200 & 290 & 77,88 & 400 & 307 \\
\hline 9 & 132,7 & 2500 & 338 & 79,64 & 450 & 357 \\
\hline 10 & 97,3 & 3000 & 300 & 85,87 & 350 & 313 \\
\hline 11 & 109,6 & 2700 & 311 & 89,06 & 350 & 322 \\
\hline 12 & 101,0 & 2400 & 247 & 101,98 & 300 & 311 \\
\hline 13 & 133,4 & 2200 & 297 & 118,92 & 300 & 367 \\
\hline 14 & 146,5 & 2200 & 324 & 112,66 & 275 & 312 \\
\hline 15 & 176,0 & 2300 & 398 & 104,90 & 350 & 365 \\
\hline 16 & 50,1 & 7000 & 366 & 32,71 & 1100 & 449 \\
\hline 17 & 54,2 & 6200 & 398 & 20,26 & 1525 & 358 \\
\hline 18 & 69,9 & 6500 & 486 & 20,95 & 2075 & 520 \\
\hline 19 & 40,6 & 9000 & 369 & 21,65 & 1750 & 448 \\
\hline 20 & 52,8 & 5700 & 310 & 30,87 & 1075 & 388 \\
\hline 21 & 64,2 & 7200 & 488 & 23,64 & 1375 & 381 \\
\hline 22 & 73,5 & 6300 & 505 & 24,20 & 1800 & 510 \\
\hline 23 & 57,1 & 7700 & 469 & 25,99 & 1425 & 410 \\
\hline
\end{tabular}

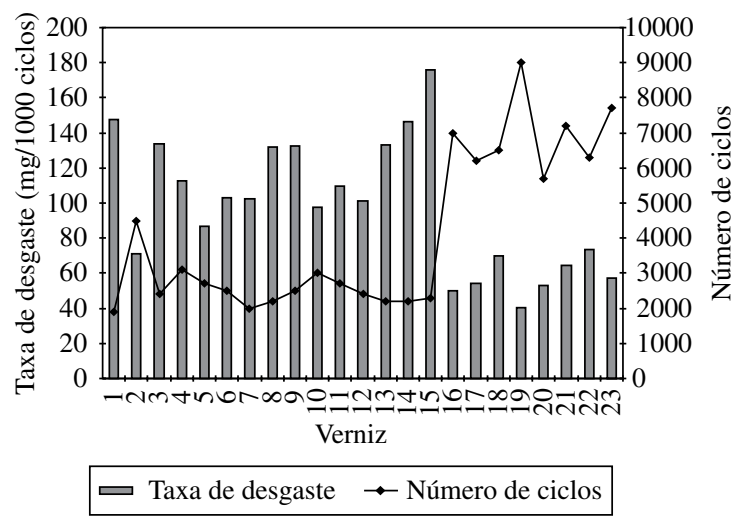

(a)

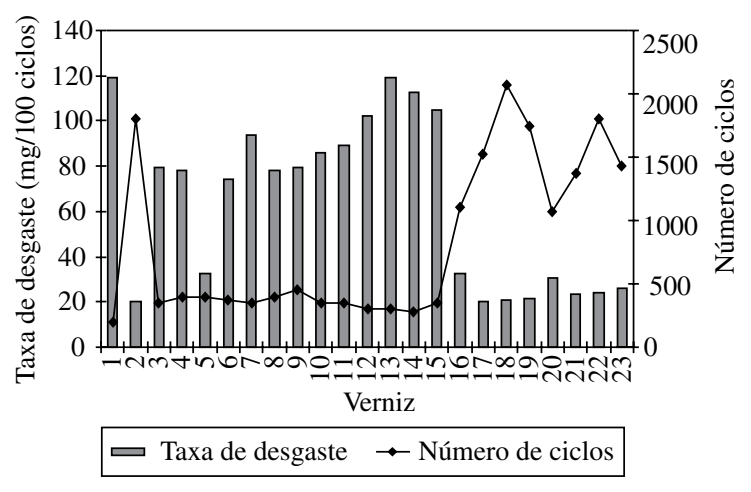

(b)

Figura 4. Gráficos de verniz x taxa de desgaste x número de ciclos para os ensaios com a) CS-17; e b) S-42.

Quando os vernizes com $10 \%$ de carga foram analisados, observou-se que o verniz com óxido de alumínio foi o que apresentou melhores resultados de resistência à abrasão, aumentando a resistência do verniz $1 \mathrm{em}$ torno de $200 \%$. O vidro \#200 aumentou a resistência à abrasão do verniz $1 \mathrm{em}$ aproximadamente $169 \%$ e o vidro \#325 e o agalmatolito apresentaram resultados próximos e aumentaram a resistência em cerca de $130 \%$. A diferença de resistência à abrasão entre os vidros \#200 e \#325 se deve ao tamanho das partículas destes, já que a sua composição é a mesma. Alguns estudos observaram que tamanhos de partículas maiores conferem ao sistema maior resistência à abrasão, já que para o desgaste de tais partículas é necessário maior esforço ${ }^{[12,13]}$.

$\mathrm{O}$ agalmatolito, apesar de ter em sua composição aproximadamente $30 \%$ de $\mathrm{Al}_{2} \mathrm{O}_{3}$, não apresentou resultados melhores do que os vidros, o que pode ser atribuído à sua morfolo- 
gia. Sabe-se que quanto mais irregular e angular a partícula, maior a resistência à abrasão; assim, quando se comparou um óxido de alumínio angular e um óxido de alumínio com partículas arredondadas, o resultado de resistência à abrasão das partículas angulares foi aproximadamente $20 \%$ maior $^{[12,13]}$. Como o agalmatolito possui uma estrutura lamelar, a sua resistência diminui e seu desgaste se dá mais facilmente.

Observou-se ainda um outro fenômeno: com o aumento da quantidade de carga no sistema, especialmente em concentrações de cargas maiores do que $15 \%$, a resistência à abrasão do filme diminui. Já foi possível constatar mediante estudos que o aumento de óxido de alumínio para quantidades maiores do que $15 \%$ não produz ganhos significativos de abrasão ${ }^{[12]}$. Além disso, com o aumento de carga no sistema, sobretudo naqueles que utilizam oligômeros uretano-acrilados em sua composição e que são considerados ligantes flexíveis - quando comparados com oligômeros epóxi-acrilados, poliéster-acrilados ou até mesmo uretano-acrilados com maior funcionalidade, como os hexafuncionais -, quanto maior a quantidade de carga e menor a quantidade de ligante, mais facilmente a carga se desprende da matriz polimérica, o que faz com que a resistência à abrasão seja menor.

Para os resultados com $\mathrm{S}-42$, verificou-se que os vernizes que obtiveram maior resistência à abrasão, ou seja, menor taxa de desgaste, mais uma vez foram aqueles em que se fizeram as combinações de óxido de alumínio e vidros, tanto \#325 quanto \#200, que são os vernizes 16 ao 23. Esses resultados também haviam sido observados nos ensaios com CS-17, mas o S-42, sendo mais agressivo, faz com que a diferença entre os resultados dos vernizes contendo misturas de cargas e do verniz contendo somente óxido de alumínio seja pequena; assim, os resultados foram muito próximos, em torno de $20 \mathrm{mg} / 100$ ciclos. O comportamento verificado nesses vernizes mais uma vez, como nos ensaios com CS-17, foi o mesmo, ou seja, os vernizes com maior quantidade de vidro apresentaram melhor resistência à abrasão. Esse fenômeno foi observado nos vernizes $17-19$ e $21-23$, dos quais os vernizes com $5 \%$ de óxido de alumínio e $5 \%$ de vidro foram os que mostraram melhor resultado, salientando-se ainda que o vidro \#200 apresentou melhor resultado do que o vidro \#325 em todos os casos. Vale lembrar mais uma vez que o ensaio com o S-42 é bastante agressivo e por essa razão perdem-se diferenças pequenas. Nota-se, assim, que os resultados nos vernizes 17 ao 19 e do 21 ao 23 ficaram bem próximos.

Quando os vernizes com 10\% de carga (vernizes 2, 3, 5 e 11) foram analisados, verificou-se mais uma vez, como no caso dos ensaios com CS-17, que o óxido de alumínio é o que apresenta melhores resultados de resistência à abrasão, aumentando a resistência do verniz 1 em cerca de 588\%. O vidro \#200 aumentou a resistência à abrasão do verniz $1 \mathrm{em}$ aproximadamente $364 \%$, mais do que nos ensaios com o CS-17, o que pode ser mais uma vez explicado pela alta agressividade dos ensaios com S-42; estes ensaios acabam desgastando rapidamente os vernizes sem carga, que, por sua vez, não oferecem grande resistência. $\mathrm{O}$ agalmatolito e o vidro \#325 mais uma vez apresentaram resultados próximos, aumentando a resistência à abrasão do verniz puro em $150 \%$.

A diferença de resistência à abrasão entre os vidros \#200 e \#325 se deve mais uma vez ao tamanho de suas partículas, conforme explicado anteriormente nos ensaios com o CS-17, e o resultado com agalmatolito se deve, como também já explicitado, à sua morfologia.

Outro fenômeno que já havia sido observado nos ensaios com o CS-17 nos vernizes de 4 a 9 e de 10 a 15: com o aumento da quantidade de carga no sistema, e neste caso sobretudo com quantidades de cargas maiores do que $20 \%$, a resistência à abrasão do filme diminui. A explicação para esses resultados já foi dada anteriormente. Nota-se, entretanto, que as diferenças nos ensaios com S-42 não são tão claras e definidas quanto aquelas nos ensaios com CS-17, isto, como já foi comentado, em decorrência da agressividade do método com S-42. Pôde-se também notar que o número de ciclos nem sempre é coerente com o valor de taxa de desgaste. Um exemplo seria o verniz 18, que apresentou taxa de desgaste um pouco inferior com relação ao verniz 17 (20,95 e 20,26 mg/100 ciclos respectivamente), porém obteve-se um resultado superior de número de ciclos, pois a camada desgastada foi maior, ou seja, o verniz 17 apresentou um resultado de 1575 para desgastar $358 \mathrm{mg}$, ao passo que o verniz 18 resistiu 2075, porém a massa desgastada foi maior e igual a $520 \mathrm{mg}$. Por essa razão vale frisar que o valor mais importante e representativo é o da taxa de desgaste.

\section{Conclusões}

Quanto aos resultados de resistência à abrasão dos vernizes desenvolvidos, os vernizes contendo vidro combinado com o óxido de alumínio apresentaram um ótimo desempenho, com resistência à abrasão superior àqueles encontrados no mercado que contém apenas o óxido de alumínio em sua composição. As misturas contendo vidro \#200 e óxido de alumínio foram as que alcançaram os melhores resultados de resistência à abrasão, especialmente aquelas com $5 \%$ de cada carga.

\section{Agradecimentos}

Os autores agradecem à Renner Sayerlack por disponibilizar o material e os equipamentos para a realização dos ensaios.

\section{Referências Bibliográficas}

1. Valera, T. S. - "Reaproveitamento de vidros laminados provenientes de rejeitos industriais e pós-consumo", Tese de Doutorado, Universidade de São Paulo, Brasil (2005). 
2. Hallensleben, M. L. - "Polyvinyl compounds, others", in: Ullmann's Encyclopedia of Industrial Chemistry, V.A21, VCH, Weinheim (1992).

3. Saunders, K. J. - "Organic polymer chemistry: an introduction to the organic chemistry of adhesives, fibres, paints, plastics and rubbers", Chapman and Hall, London (1988).

4. Boyd, D. C.; Danielson, P. S. \& Thompson, D.A. - "Glass" in: Kirk-Othmer, Encyclopedia of Chemical Technology, V.12, Ed. Wiley Interscience, USA (1994).

5. Varshneya, A. K. -“Fundamentals of inorganic glasses”, Academic Press,USA (1994).

6. Holzheim, R. -“Empresa inicia programa para incentivar reciclagem", Disponível em: <http://www.setorreciclagem.com.br>.

7. Bleus, J. P et al. - European Coatings Journal, p.30-34, jan/fev (2005).

8. Biesbrouck, K. - "Hard or flexible coatings: influence on measured performances" in: Radtech Europe 2005, 12., Conference Proceedings, Barcelone, Spain, CDROM (2005).
9. Barbeau, P.; Muzeau, E. \& Hirsch-askienazy, A. - "The mechanical performances of urethane acrylate oligomers" in: Radtech Europe 99, 9., Conference Proceedings, Berlin, Germany (1999).

10. Koleske, J. V. - Journal of Coatings Technology, 69, p.29-38 (1997).

11. Bauer, F. et al. - "Nano/Micro particle reinforced polyacrylates for flooring applications" in: Radtech Europe 2005, 12., Barcelone, Spain. Conference Proceedings, CD-ROM (2005).

12. Menzel, K.; Biehler, M. \& Lokai, M. - "Formulation of $U V$-parquet coatings with respect to adhesion, abrasion and scratch resistance" in: Anais do $9^{\circ}$ Congresso Internacional de Tintas, São Paulo (2005).

13. Flyunt, R. et al. - "Development of Scratch and Abrasion resistant $U V$-coatings for wood surfaces based on solvent-poor nanocomposite lacquers" in: $5^{\text {th }}$ Wooodcoatings Congress, Prague, Czech Republic, Congress Papers (2006).

Enviado: 16/12/06

Reenviado: $27 / 02 / 07$

Aceito: $14 / 03 / 07$ 